\title{
Natural Supramolecular Building Blocks: Icosahedral Virus Particles Organized by Attached Oligonucleotides
}

\author{
Erica Strable, John E. Johnson, ${ }^{*}$ and M.G. Finn* \\ Departments of Chemistry and Molecular Biology, and The Skaggs Institute for Chemical Biology \\ The Scripps Research Institute, 10550 N. Torrey Pines Rd., La Jolla, CA 92037
}

\section{Supporting Information}

\section{Preparation of DNA-Virus conjugates}

All oligonucleotides used in this paper were purchased from Operon Technologies. Complementary 20-mer oligonucleotides functionalized at the $5^{\prime}$-end with NHS ester or maleimide groups were prepared and attached to the appropriate CPMV scaffold (wild-type for NHS esters and Cys mutant for maleimides) as shown in Figure S1. Coupling of 0.4 umole of the amine terminated oligo to the bifunctional cross linker were done using a 10-fold excess of sulfo-SMCC (Pierce) or a 50 -fold excess of Water soluble DSP (Pierce) in $1 \mathrm{~mL}$ of $0.1 \mathrm{M}$ potassium phosphate buffer $\mathrm{pH} 7.0$ for 6 hours at room temperature. The oligo-linker conjugates were purified from the excess linker using a HighTrap-Q column (Amersham) on an Akta Explorer (Amersham). Figure S2 shows a sample purification to isolate the oligo-linker conjugate.

In each case, the activated oligonucleotide reactants were incubated at $4^{\circ} \mathrm{C}$ in varying molar ratios with respect to viral protein (up to 50:1) to obtain derivatized virions with different degrees of oligonucleotide loading. Purification was accomplished by ultracentrifugation pelletting through a sucrose cushion to separate intact particles from the excess small molecules. The yields of recovered virus ranged from $75 \%$ to $90 \%$, and the resulting materials were shown to be composed of intact particles by sucrose gradient sedimentation and the observation of characteristic $A_{260} / A_{280}$ ratios (approximately 1.7). Furthermore, size-exclusion chromatography (Superose-6) at $\mathrm{pH} 7$ showed no protein or nucleic acid fragments that would arise from disassembled virions. ${ }^{1}$ These results confirm that there is little or no disassembly of the virus during the coupling process.

The extent of covalent attachment of oligonucleotide to the virus surface was determined with the use of fluorescein-labeled oligonucleotides. Measurement of dye absorbance (495 nm) relative to that of the virus $(260 \mathrm{~nm})$ has been used routinely for quantification of the direct attachment of dyes to the viral capsid. ${ }^{2-4}$ Thus, wild-type CPMV conjugate WT・N1FI showed an average of $30 \pm 3$ single-stranded oligonucleotides attached per capsid, while EF-Cys•S1FI displayed $30 \pm 1.5$ strands per capsid. We assume, on the basis of excellent reproducibility observed with WT•N1FI and EF-Cys•S1FI, that the conjugates WT•N1, WT•N2, EF-Cys•S1, and EF-Cys•S2, which lack fluorescein, bear the same number of oligonucleotides as the dye-labeled conjugates. These loading values are approximately the same as those obtained with simple dyeNHS ester and dye-maleimide reagents that attach to the particle surface under otherwise identical conditions; ${ }^{3,4}$. The distributions of the numbers of attached oligonucleotides per virion is quite narrow, as judged from the appearance of the anion-exchange FPLC peaks shown below, suggesting that the reaction is well-behaved, albeit slow. Below, the number of attached oligonucleotides is denoted after the compound number for each virus; thus, EF-Cys $\mathbf{S} 1_{15}$ represents the EF-Cys virus bearing an average of $15 \mathrm{~S} 1$ oligonucleotides per particle. 
Figure S1.

Preparation of CPMV-

oligonucleotide

samples. The

designation for each

DNA derivatized virus

particle incorporates

the type of virus,

linker, and

oligonucleotide used.

Viruses labeled $\mathbf{1}$ and

$\boldsymbol{1 F} \boldsymbol{l}$ contain the

complementary

oligonucleotide

sequence to 2 and

$2 F l$.

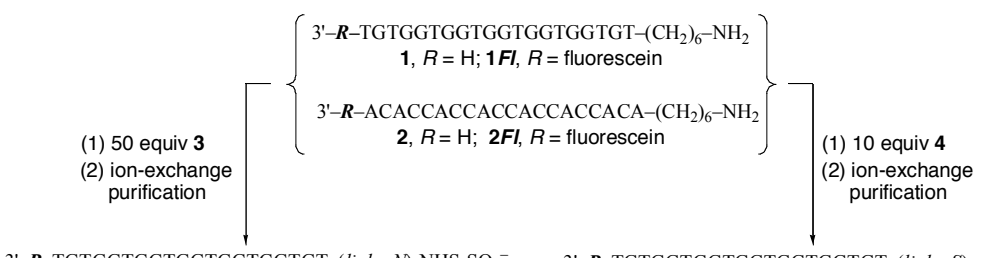

3'- $\boldsymbol{R}$-TGTGGTGGTGGTGGTGGTGT-(linker $\mathrm{N}$ )-NHS-SO ${ }_{3}^{-}$ N1, $R=\mathrm{H} ; \mathbf{N} 1 F I, R=$ fluorescein

3'- $\boldsymbol{R}$-TGTGGTGGTGGTGGTGGTGT-(linkerS)-maleimide $\mathbf{S 1}, R=\mathrm{H} ; \mathbf{S} 1 F I, R=$ fluorescein

3'- $\boldsymbol{R}$-ACACCACCACCACCACCACA-(linker $N$ )-NHS-SO ${ }_{3}^{-} \quad$ 3'- $\boldsymbol{R}$-ACACCACCACCACCACCACA-(linker $S$ )-maleimide N2, $R=\mathrm{H} ; \quad \mathrm{N} 2 \mathrm{FI}, R=$ fluorescein S2 $R=\mathrm{H} ;$ S2FI, $R=$ fluorescein
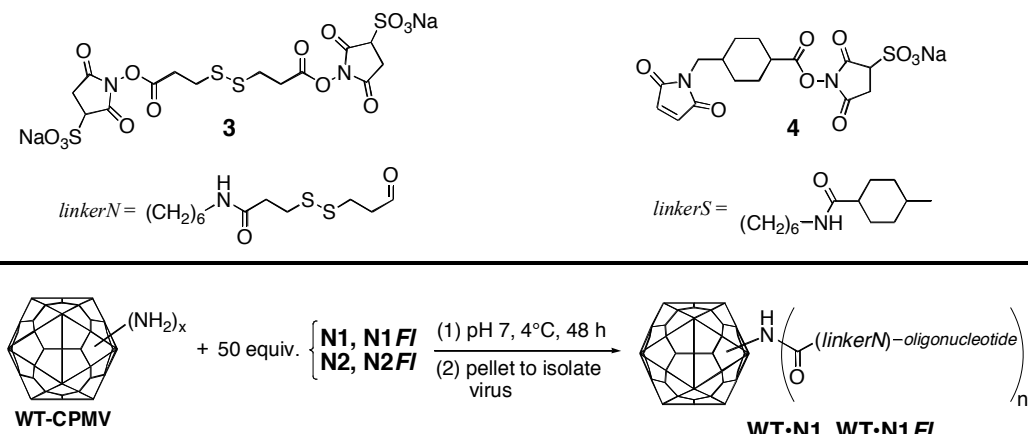

WT·N1, WT·N1FI $\mathrm{WT} \cdot \mathrm{N} 2, \mathrm{WT} \cdot \mathrm{N} 2 \mathrm{FI}$ $\mathrm{n}=10 \pm 1$
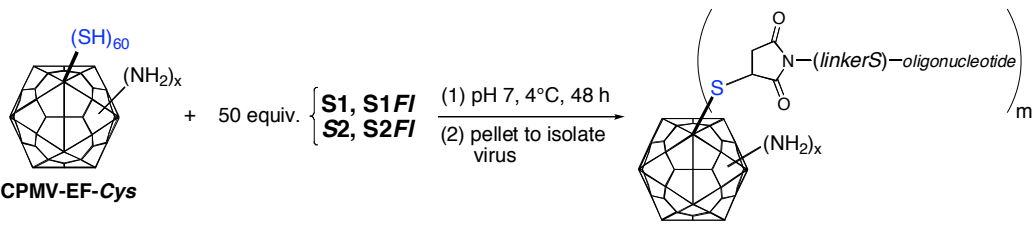

$\mathrm{EF}-$ Cys.S1, EF-Cys.S1FI $\mathrm{EF}-\mathrm{Cys} \cdot \mathrm{S} 2$, EF-Cys.S2FI $\mathrm{m}=15 \pm 1.5$

Figure S2. Representative purification of reactive oligonucleotide conjugate (N1) on a $5 \mathrm{~mL}$ High Trap-Q colunmn, eluting with $0.1 \mathrm{M} \mathrm{K}$ phosphate (pH 7.0) and then stepping to $1 \mathrm{M} \mathrm{NaCl}$ in that buffer at $110 \mathrm{~min}$. Note that a large volume of low ionic-strength buffer is required to get clean separation of the excess linker from the desired product.

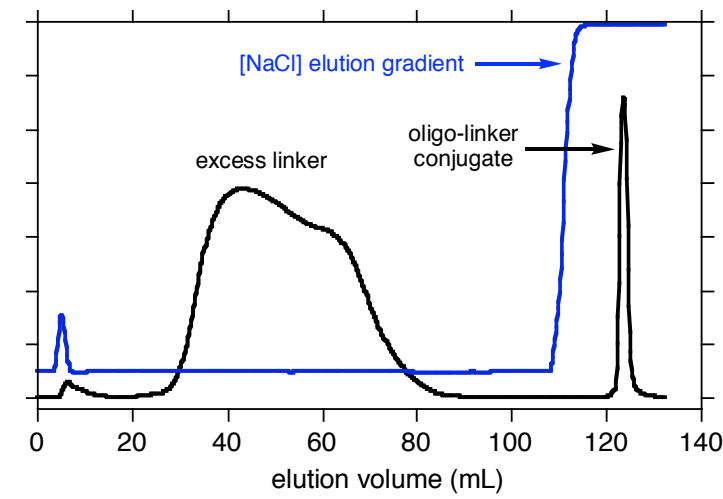

Anion-exchange FPLC is highly sensitive to the number of attached oligonucleotide polyanions, as shown in Figure S3. With a standard elution protocol, excellent resolution is observed among underivatized virions and those bearing an average of 4,15 , and 30 
oligonucleotides, with an increasing loading giving rise to greater retention time, consistent with an increase in the anionic charge on the particle. SDS-PAGE analysis of the derivatized cysteine mutant shows a shift and broadening of the band corresponding to the large protein subunit, with the small subunit band remaining unperturbed. This is consistent with the denatured large subunit being composed of a mixture of normal (42 kD) and oligonucleotide-conjugated (48 kD) proteins, the latter showing a slight retardation in electrophoretic mobility consistent with its increased molecular weight. Since the reactive cysteine of CPMV-EF-Cys is located on the large subunit, oligonucleotide derivatization is found exclusively on that component.

Figure S3. Characterization of oligonucleotide-decorated CPMV. (Left) Anion-exchange FPLC of (A) separate injections of WT-CPMV (black) and EF-Cys $\bullet \mathbf{S}_{24}$ (red), (B) EF-Cys $\bullet \mathbf{S ~ 2} \mathbf{1}_{15}$, (C) a mixture of the three species; and (D) separate injections of WT-CPMV (black), EF-C $\boldsymbol{y} \boldsymbol{s} \bullet \mathbf{S}_{\mathbf{2 4}}$ (purple), and EF-Cys $\mathbf{S}_{30}$ (red). (Right) SDSPAGE: lane 1, MW ladder; lane 2, WT-CPMV; lane 3, EF-Cys $\bullet \mathbf{S 2} \mathbf{1}_{15}$. The arrow marks the shifted band for the large CPMV subunit protein; the band at higher molecular weight in lane 3 is present in the EF-Cys virus prior to oligonucleotide attachment.
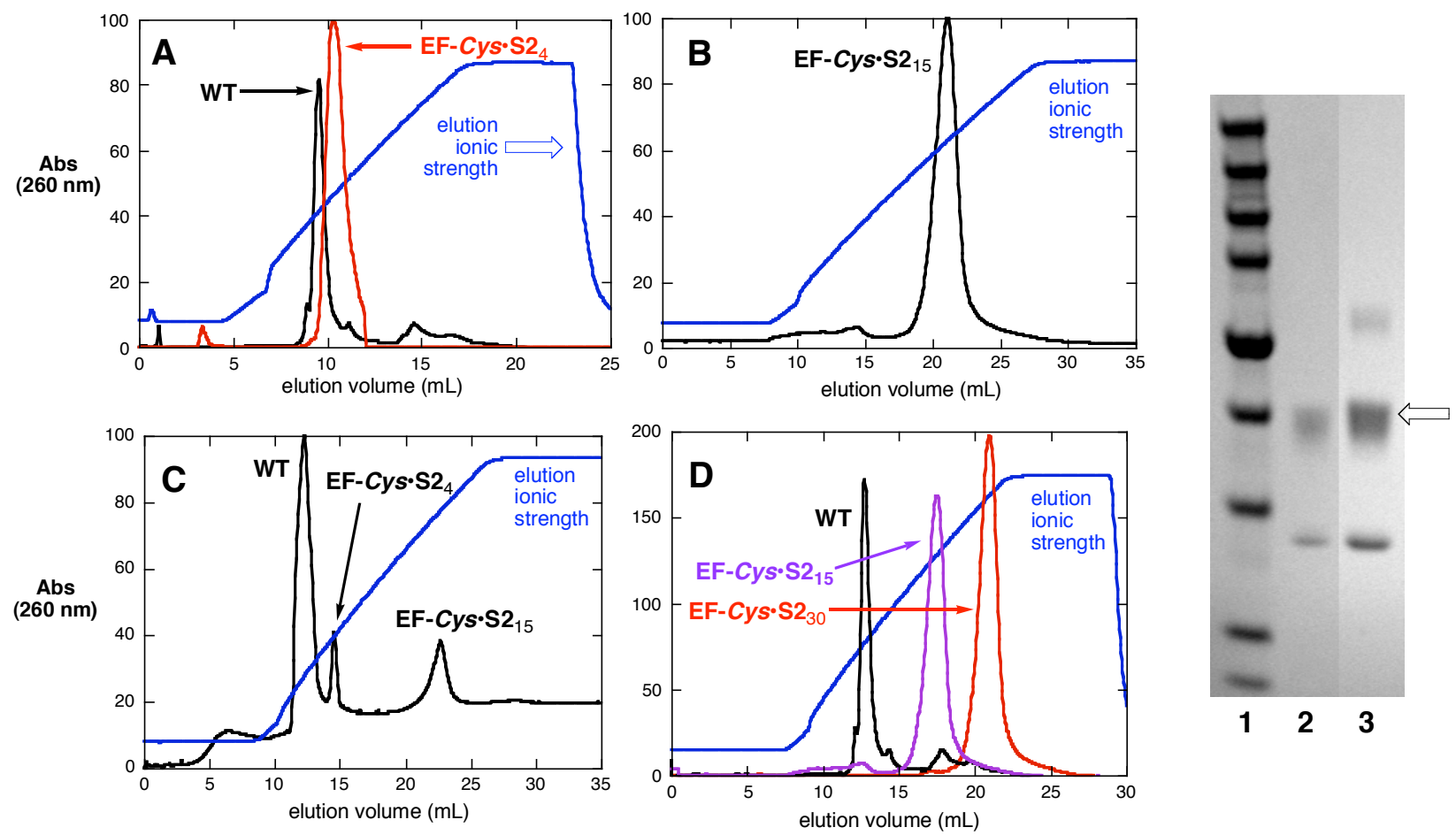

Multi-angle dynamic light scattering was used to provide an independent measure of the molecular weights and average hydrodynamic radii of derivatized particles in the solution phase. Wild-type CPMV was found by this technique to have a molecular weight of $6.33 \pm 0.1 \times 10^{6}$ Daltons and a radius of $13.3 \pm 0.8 \mathrm{~nm}$, in good agreement with known values. Samples of $\mathbf{W T} \cdot \mathbf{N} 1_{10}$ (Figure S4) and EF-Cys.S2 ${ }_{15}$ (data not shown) eluted on size-exclusion chromatography as two peaks at retention times shorter than wild-type CPMV, indicating an increase in the effective size of the particle. Furthermore, "peak 2" (Figure S4) gradually converts to "peak 1" upon storage in buffer containing $1 \mathrm{M} \mathrm{NaCl}$ solution. These data suggest that oligonucleotide-directed aggregation occurs via non-Watson-Crick base pairing and not by disulfide bond formation between particles. We therefore assign "peak 2" to the single derivatized virion, and "peak 1" to a dimeric aggregate. Aggregates larger than this, such as the ones described below, cannot enter the size-exclusion column. 
Figure S4. Comparison of wild-type CPMV and $\mathbf{W T} \cdot \mathbf{N} \mathbf{1}_{\mathbf{1 0}}$ by dynamic light scattering coupled to a size exclusion column (Superose 6). The values of molecular weight and mean hydrodynamic radius obtained by light-scattering measurements of the eluted fractions are shown.

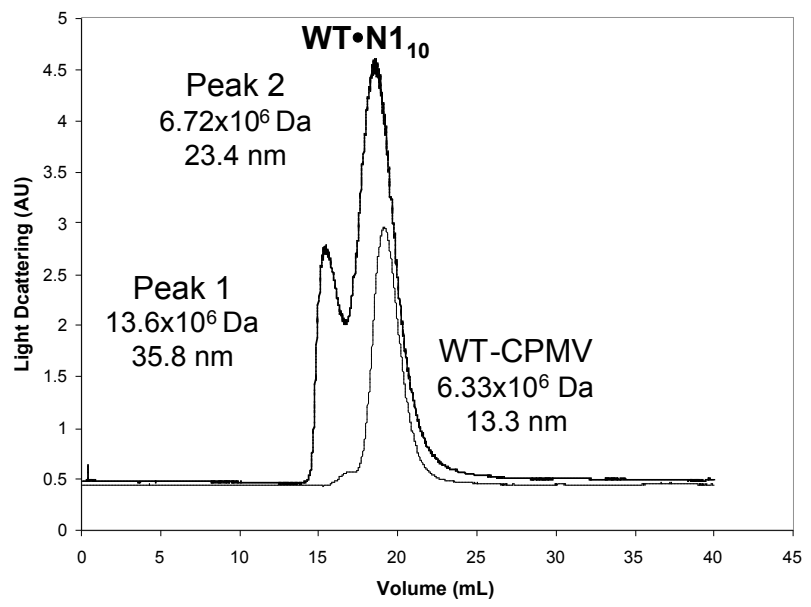

Single particles of $\mathrm{WT} \cdot \mathrm{N} 1_{10}$ were found to have molecular weights of $6.72 \pm 0.1 \times 10^{6} \mathrm{Da}$, a measured increase of approximately $390 \mathrm{kDa}$, in reasonably good agreement to the expected value of $70 \mathrm{kDa}$ from the addition of 10 oligonucleotide chains. The magnitude of the increase in hydrodynamic radius (13.8 to $23.4 \mathrm{~nm}$ ) is roughly equivalent to the length of a helical 28-mer strand of duplex DNA. However, the appended single-stranded 20-mer oligonucleotides are not likely to assume such a conformation, but instead should be highly flexible, and the hydrodynamic radius is likely to be sensitive to the chemical nature of the surface of the particle and its interaction with solvent. Thus, an increase in hydrodynamic radius is difficult to interpret in detail, but it is clear that the CPMV particle is altered significantly by oligonucleotide attachment. While multi-angle dynamic light scattering has been used to characterize species such as polysaccharides, ${ }^{5-7}$ lignins, ${ }^{8}$ and other biopolymers, ${ }^{9-11}$ it has not previously been applied to virus particles.

When CPMV particles bearing complementary oligonucleotide sequences were mixed in solution, an immediate increase in absorbance at $320 \mathrm{~nm}$ was noted, presumably resulting from increased light scattering by aggregates (Figure S5). At room temperature, aggregation was complete by this measure within approximately one hour. The subsequent addition of excess oligonucleotide 1 or 2 (with or without the aminohexyl linker at the $5^{\prime}$ end) resulted in a decrease in $A_{320}$ of the mixture back to the original value, consistent with the disruption of duplexoligonucleotide aggregates by competing soluble oligonucleotide.

Figure S5. $A_{320}$ vs. time as a measure of virus aggregation following mixing of components. (a) EF-Cys $\bullet \mathbf{S} \mathbf{1}_{15}+\mathbf{E F}$ $C y s \cdot S 2_{15}$, (b) EF-Cys $\bullet \mathrm{S1}_{15}+\mathrm{EF}-\mathrm{Cys}$, (c) EF-Cys $\bullet \mathrm{S1}_{15}+\mathrm{WT}-\mathrm{CPMV}$, (d) repeat of (a), followed by addition of 500 equiv. of oligonucleotide 1 at the time marked by the arrow.

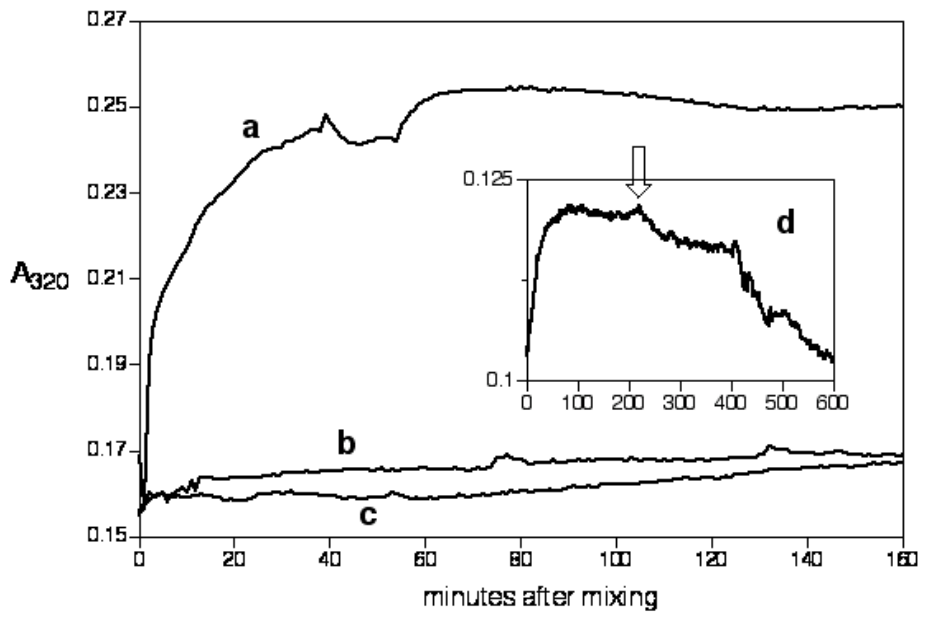


Transmission electron microscopy (TEM) analysis of mixtures involving complementary oligonucleotide sequences revealed sequence-specific aggregation of virions into well-ordered arrays, shown in Figure S6. Thus, mixtures of virus particles bearing complementary sequences at $4^{\circ} \mathrm{C}$ or room temperature assembled into arrays that appear to be hexagonally close-packed in two dimensions (Figure S6B-D). The three-dimensional nature of these assemblies in solution is uncertain, since TEM provides little information on this score, but we see no reason why three-dimensional order should be disfavored. Indeed, upon prolonged incubation, all of the virus in such mixtures precipitated from solution, even at relatively low initial concentrations $(0.5 \mathrm{mg} / \mathrm{mL})$, presumably due to the formation of large aggregates. No aggregates were formed when the virus particles did not bear complementary sequences. The observed arrays adopted the same appearance in TEM analysis for each of the possible complementary combinations: [WT•N1 + WT・N2], [WT•N1 + EF-Cys・S2], [EF-Cys・S1 + WT•N2], and [EF-Cys•S1 + EF-Cys•S2]. That both wild type and mutant viruses behave similarly is unsurprising in light of the fact that the reactive centers for these virions (Lys38 and the inserted cysteine, respectively) are separated by only approximately $10 \AA$ (Fig. 1).

The oligonucleotide-mediated aggregates were sundered into individual virus particles by treatment with excess complementary oligonucleotide (Fig. 8E) or DNase (Fig. 8I), but not by treatment with non-complementary oligonucleotide $(8 \mathrm{~F})$, high salt concentration $(8 \mathrm{G})$, RNase $(8 \mathrm{H})$, or dilution to low overall protein concentration $(0.05 \mathrm{mg} / \mathrm{mL}$, data not shown). These data demonstrate that the anticipated specific duplex base pairing holds the aggregates together. Furthermore, only the aggregates involving viruses $\mathbf{W T} \cdot \mathbf{N} \mathbf{1}_{10}$ and $\mathbf{W T} \cdot \mathbf{N} \mathbf{2}_{10}$ were disrupted by treatment with dithiothreitol, by reduction of the disulfide bond present in the linker used to prepare these conjugates.

The sharp thermal melting behavior of oligonucleotide-virus aggregates at approximately $45^{\circ} \mathrm{C}$ (Figure 5) was reproduced for the particles bearing 15 or 30 oligonucleotides per virion. More than $90 \%$ of unarrayed oligonucleotide-labeled particles or unmodified particles remained intact when heated to $45^{\circ} \mathrm{C}$ for the same amount of time. 
Figure S6. Self-organization of CPMV by oligonucleotide complementation. (A) Schematic representation of the phenomenon. (B-D) Aggregation of the following mixtures $(1 \mathrm{mg} / \mathrm{mL}$ total virus) at room temperature for 16 hours: (B) $\mathbf{W T} \cdot \mathbf{N} 1_{10}+\mathbf{W T} \cdot \mathbf{N} \mathbf{2}_{10}, \quad$ (C) $\mathbf{E F}-$ $C y s \cdot S 1_{15}+\mathrm{EF}-C y s \cdot S 2_{15}$, and (D) $\mathrm{EF}-\mathrm{Cys}_{\boldsymbol{N}} \bullet \mathrm{S}_{15}+\mathrm{EF}-\mathrm{Cys}_{15} \bullet \mathrm{S} 2_{15}$. Note that in these samples, most of the virus particles were found to be aggregated, in contrast to all negative control samples at the same concentration, in which most of the virus particles were dispersed and the few clusters observed were randomly organized. The aggregates were shown to be directed by oligo-deoxynucleotide base-pairing interactions by the following experiments, in which preformed aggregates were exposed to the following: (E) 100-fold excess of soluble complementary oligonucleotide (with respect to viralbound oligonucleotide); (F) 100-fold excess of noncomplementary oligonucleotide 5 ACTGGTCAACTGGTCAACCTG; (G) $1 \mathrm{M} \mathrm{NaCl}$; (H) RNase A; (I) DNase 1.
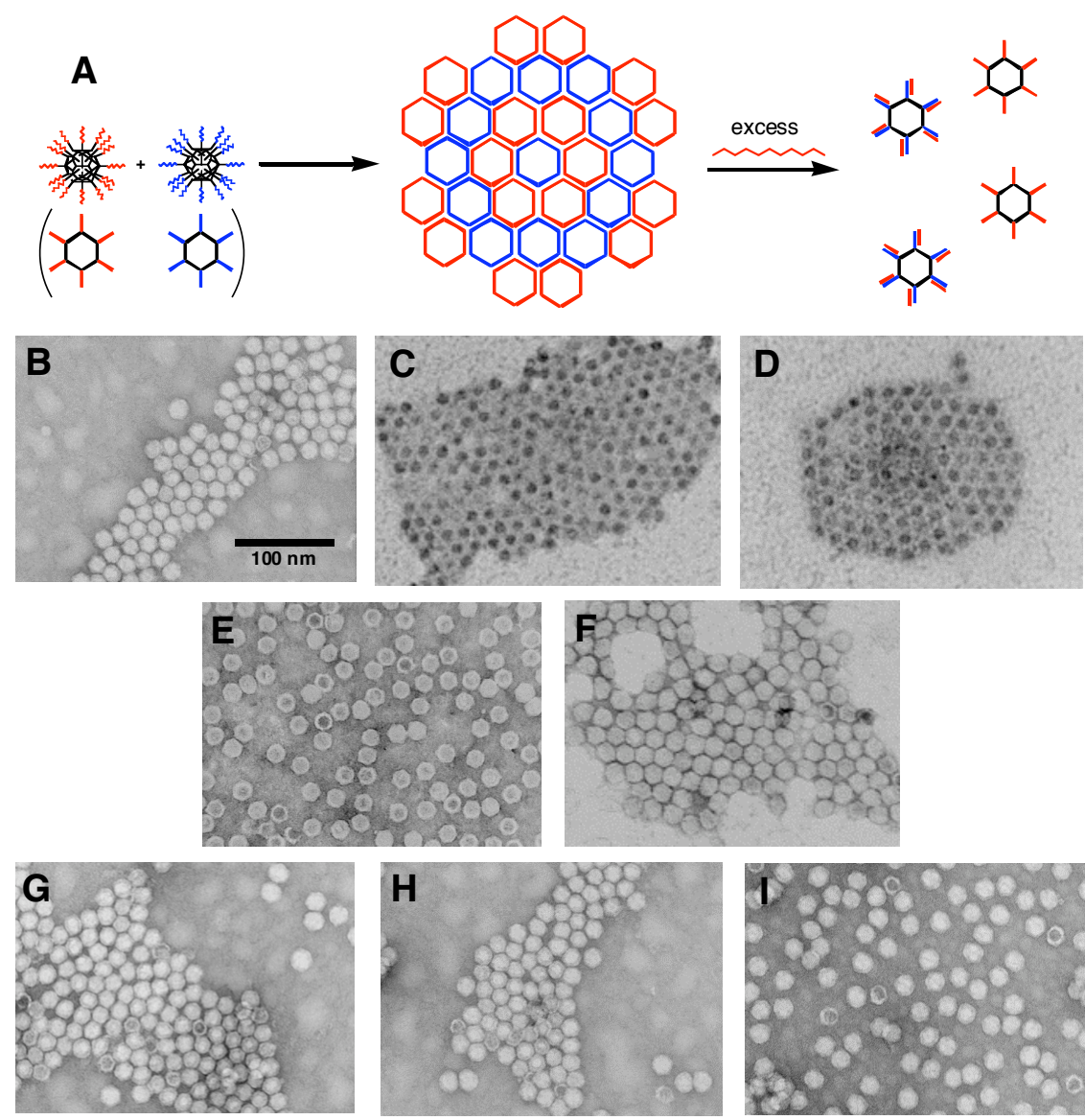


\section{References and Notes.}

1. At pH 3.5, oligonucleotide-decorated CPMV particles are somewhat less stable than the wildtype virus, as revealed by the observation of peaks on size-exclusion FPLC at later retention times (RNA and disassembled protein subunits) and a dearth of intact particles visible by TEM.

2. Wang, Q.; Lin, T.; Tang, L.; Johnson, J. E.; Finn, M. G. Angew. Chem. Int. Ed. 2002, 41, 459462.

3. Wang, Q.; Kaltgrad, E.; Lin, T.; Johnson, J. E.; Finn, M. G. Chem. Biol. 2002, 9, 805-811.

4. Wang, Q.; Lin, T.; Johnson, J. E.; Finn, M. G. Chem. Biol. 2002, 9, 813-819.

5. Girod, S.; Baldet-Dupy, P.; Maillols, H.; Devoisselle, J. M. Journal of Chromatography, A 2002, $943,147-152$.

6. Kulicke, W.-M.; Lettau, A. I.; Thielking, H. Carbohydrate Research 1997, 297, 135-143.

7. Vaarum, K. M.; Smidsroed, O.; Brant, D. A. Food Hydrocolloids 1992, 5, 497-511.

8. Fredheim, G. E.; Braaten, S. M.; Christensen, B. E. Journal of Chromatography, A 2002, 942, 191-199.

9. Wittgren, B.; Wahlund, K. G. Carbohydrate Polymers 2000, 43, 63-73.

10. Mendichi, R.; Giammona, G.; Cavallaro, G.; Giacometti Schieroni, A. Polymer 2000, 41, 86498657.

11. Ioan, C. E.; Aberle, T.; Burchard, W. Macromolecules 2000, 33, 5730-5739. 